\title{
La recepción francesa de Hegel en las pos- trimerías del S.XX: Spinoza y la tradición neoplatónica*
}

\section{The French Reception of Hegel in the Late 20th Century: Spinoza and Neoplatonism}

RESUMEN: Para Hegel, la substancia de Spinoza es el comienzo necesario de toda filosofía, pero es solo su comienzo, pues carece de la libertad de la subjetividad. Los críticos materialistas franceses -Althusser, Macherey, Deleuze- señalaron que Hegel había reducido el sistema de Spinoza a una mera variante de la ontología jerárquica neoplatónica. El autor sostiene que estos mismos críticos redujeron a Hegel a una simple inversión del orden neoplatónico. Esta mala interpretación mutua, en todo caso, resulta ser productiva en la medida en que gira en torno a temas clave: la crítica de la filosofía de la identidad, el rechazo a la causalidad emanativa y el concepto de la diferencia primordial. El autor propone entenderlos como la cuestión central de la negatividad.

PALABRAS CLAVE: SUSTANCIA - SUJETO, - NEGATIVIDAD - DIFERENCIA - ONTOLOGÍA
ABSTRACT: For Hegel, Spinoza's substance is the necessary beginning of all philosophy, but only its beginning, because it lacks the freedom of subjectivity. The French materialist critics - Althusser, Macherey, Deleuze - pointed out that Hegel reduced Spinoza's system to a mere variation of Neoplatonic hierarchical ontology. The author argues that these critics themselves reduced Hegel to a simple inversion of Neoplatonic order. This mutual misreading nevertheless proves to be a productive one insofar as it revolves around key topics: critique of the philosophy of identity, rejection of emanative causality, and a concept of primordial difference. The author proposes to grasp these as the central question of negativity.

KEY WORDS: SUBSTANCE, SUBJECT, NEGATIVITY, DIFFERENCE, ONTOLOGY

\footnotetext{
*Traducción de Narciso López

(C) Studia Hegeliana, vol. II (2016), pp. 127-140. ISSN: 2444-0809

Sociedad Española de Estudios sobre Hegel
} 


\section{LA POLÉMICA}

Si tuviéramos que elaborar una lista con los principales interlocutores filo$\checkmark$ sóficos de Hegel, con sus adversarios favoritos, nos veríamos obligados a situar la filosofía de Spinoza muy cerca del primer puesto. Todos los clásicos del idealismo alemán la discutieron e incluso puede que no sea demasiado exagerado afirmar que no habrían podido desarrollar sus respectivos sistemas filosóficos sin reflejarse en el espejo del spinozismo (Macherey 2011, 195). Si es cierto que, en general, la postura de la primera etapa de Hegel era spinozista (Düsing 1992, 164), esto solo fue posible porque existía por aquel entonces una imagen estereotípica del spinozismo que exigía un compromiso inmediato, ya a favor o en contra. ¿Pero por qué Spinoza, un pensador de la naturaleza que carecía de una deidad trascendente, se convirtió de repente en un tema de discusión general y, en cierta medida, en un filósofo ejemplar, en el filósofo? La respuesta reside, en su mayor parte, en una larga polémica que mantuvieron algunos destacados intelectuales y que se conoce como Pantheismusstreit o Spinozismusstreit.

La correspondencia que Jacobi mantuvo con Mendelsohn fue la chispa que desató esta polémica en la que terminó involucrándose toda la élite intelectual del momento, desde Herder y Hamman, hasta Reinhold y Kant. En ella, Jacobi sostuvo que si la perspectiva del solo entendimiento se llevaba hasta sus últimas consecuencias, esta desembocaba en el determinismo y el fatalismo y era, por tanto, fundamentalmente inmoral. Para él, el spinozismo era la forma más radical y a la vez la más congruente de un sistema racional. Por eso afirmó que una filosofía basada en el entendimiento es necesariamente una forma de spinozismo. De ahí, la alternativa: o bien uno es un filósofo y, por tanto, un spinoziano, o bien uno ha de rechazar el spinozismo y con ello los principios del entendimiento discursivo y la filosofía. El alcance del conocimiento que se podía llegar a obtener por medio del entendimiento y la filosofía era demasiado corto para Jacobi. Según él, estos no podían captar el núcleo de la verdad y se limitaban a formular enunciados verdaderos. Este seguía siendo, no obstante, algo inmediato e inanalizable, algo que solo podía captarse por una intuición de fe. Todo conocimiento verdadero debía estar, por tanto, fundado en la fe.

Podemos utilizar estas tesis de Jacobi como trasfondo negativo sobre el que formular los desafíos fundamentales a los que tuvo que enfrentarse el idealismo alemán: el de cómo garantizar y defender el lugar ético de la libertad en el marco de la filosofía como "spinozismo"; o en otro contexto: el de dirimir si el conocimiento necesita una garantía externa -como la fe o la intuición- o si, por el contrario, está garantizado intrínsecamente como conocimiento, al ser capaz, tal vez, de producir su propio fundamento. Esto fue lo que llevó a los 
idealistas alemanes a abrazar el spinozismo como un sistema filosófico ejemplar, mientras intentaban refutarlo y reemplazarlo al mismo tiempo ${ }^{1}$.

\section{El Spinoza DE Hegel}

Por un lado, Hegel afirmó que Spinoza era el pináculo de la filosofía moderna, si acaso el único comienzo posible en filosofía; incluso llegó a sostener que no hay ninguna filosofía salvo el spinozismo ${ }^{2}$ (TWA20, 157-159). Por otro lado, la mayoría de los reproches de Hegel a Spinoza pueden resumirse en el reproche de que esa misma filosofía que da lugar a la posibilidad de la filosofía como tal nunca fue más allá de su punto de partida, jamás desarrolló sus propias posiciones. En tanto que era incapaz de pensar la contradicción, Hegel la entendió a menudo como un principio parmenídeo o de "identidad", según el cual solo el ser es, mientras que el no-ser no es; la consideraba un fósil viviente para reintroducir, supuestamente, una filosofía de la luz, no cristiana, "oriental", así como el principio ex nihilo nihil fit en la filosofía. El propio Hegel defendió enérgicamente la metafísica cristiana y su principio de creatio ex nihilo ( $C L$, 226-227).

El reproche de Hegel a la inmovilidad y rigidez de Spinoza puede analizarse de tres maneras diferentes, aunque íntimamente relacionadas. En primer lugar, Hegel sostuvo que la substancia spinoziana era incapaz de transformarse a sí misma o de crecer orgánicamente. Por decirlo según la formulación decisiva de Hegel, era una substancia que todavía no era substancia y sujeto. A este respecto, el spinozismo era una variante del "panteísmo de los eleáticos" (CL, 227), y su substancia era inmóvil exactamente en la misma medida en la que lo era el ser de los eleáticos: era una pura afirmación e inmediatez abstractas que no implicaba movimiento ni contradicción. En segundo lugar, el principio de demostración more geometrico de Spinoza era el método de demostración de las matemáticas que tanto Hegel como otros idealistas alemanes desecharon en virtud de su incapacidad para captar la naturaleza que se autodespliega y el movimiento orgánico del absoluto. En este sentido, la rigidez de la substancia se encuentra íntimamente relacionada con la rigidez del método con el que se explica (TWA20, 161). En tercer lugar, pese a que el spinozismo había logrado formular los más brillantes conceptos dialécticos, fracasó a la hora de desarrollarlos hasta sus conclusiones últimas. Según Hegel, Spinoza ya había formulado en la definición de causa sui "la no diferencia entre el ser y la nada"; ya había captado en este concepto la idea especulativa fundamental de la automediación -puesto que la causa de sí se produce a sí misma como su efecto y, por tanto,

[1] Véase Vaysee 2007, 65; Tavoillot 2007, 40.

[2] La alternativa entweder Spinozismus oder keine Philosophie suena parecida a la alternativa propuesta por Jacobi ( $c f$. también Johnston 2014, 34). 
como algo otro de sí misma- pero, en apariencia, fracasó a la hora de aplicar estos principios a la substancia absoluta, ya que, de otro modo, esta no habría sido inmóvil (TWA20, 168).

¿Qué tipo de lectura hizo Hegel de la filosofía de Spinoza? Sería demasiado ingenuo -cuando no completamente erróneo- decir que seleccionaba algunos conceptos spinozianos y los introducía en su propia filosofía, al tiempo que descartaba otros. Muy al contrario, la lectura hegeliana nunca deja de admitir que el texto que está leyendo es una expresión necesaria del espíritu y que, por tanto, ya está en sí mismo en la verdad: no es solo una colección más o menos afortunada de afirmaciones exitosas y fallidas. Se trata de una lectura que no mide su texto en relación a un patrón externo, sino que se empeña en llevar a cabo una explicación inmanente. Por lo tanto, un texto ya está siempre en la verdad; pero, al mismo tiempo, su verdad nunca es una verdad completa, absoluta o plena. Es como si la verdad expresada en el texto se convirtiese, por medio de esta misma expresión, en algo distinto de lo que era, exigiendo, por tanto, una nueva expresión. Un texto como expresión del espíritu ya implica algo que no puede ser captado en su forma inmediata; implica algo no expresado. Es por eso por lo que la lectura hegeliana persigue repetir el texto en su verdad; es una repetición productiva de la verdad del texto que está leyendo ${ }^{3}$.

Podemos formular la cuestión que interesó a Hegel de Spinoza como la cuestión de si es posible pensar la contradicción o el movimiento en el nivel de la substancia absoluta o, por decirlo con Žižek, si es posible pensar el absoluto como un absoluto frágil (Žižek 2001). Dicho con más exactitud: es la cuestión de cómo puede uno leer y explicar el spinozismo con el fin de producir con éxito un concepto tal del absoluto. Lo que Hegel encontró en Spinoza era la idea de que la substancia era una y universal, pero también halló la idea de que toda determinación particular ya es una negación de esta unidad primordial. Lo que Hegel no encontró en Spinoza era algo no del todo ajeno a esa filosofía , algo que tuviese que ser añadido a ella artificialmente, desde el exterior; sino algo que, ciertamente, ya estaba allí, escrito en la primera línea de la primera parte de la obra más importante de Spinoza, la Ética, en la definición de la causa sui. La lectura especulativa de Hegel no considera que el spinozismo sea su adversario, sino que, estrictamente hablando, lo considera una parte integrante de su propia postura ${ }^{4}$. Lo que Hegel echaba en falta en Spinoza era una forma de insistencia: si Spinoza hubiera entendido la causa de sí como autodeterminación

[3] La lectura hegeliana pone en marcha todos los aspectos necesarios para el desarrollo de la hermenéutica gadameriana, en la cual la lectura se entiende como lectura religiosa, esto es, re-lectura (re-legere) que tiene como objetivo resucitar «el rastro muerto del significado» (Gadamer 2004, 156).

[4] Véase también Düsing 1992, 163. 
de la substancia universal, ya habría tenido a su disposición una formulación explícita de la negación de la negación, un concepto de contradicción productiva $y$, por tanto, un concepto de un absoluto en movimiento.

Así pues, la cuestión de Hegel sobre la filosofía de Spinoza es una cuestión de movimiento o contradicción del comienzo, de lo primordial; es una cuestión del dinamismo interno del ser mismo; es una cuestión sobre por qué el spinozismo tuvo que quedarse atascado en el principio, en la primera frase, sobre por qué fue incapaz de moverse desde este comienzo. Por utilizar una fórmula recurrente: ¿por qué al spinozismo le pareció que su absoluto estaba inmóvil, mientras que a la lectura hegeliana le parecía que ya era un absoluto con dinamismo interno?

\section{MALENTENDIDOS PRODUCTIVOS}

Antes de sumergirme más profundamente en la lectura de Spinoza que hace Hegel, señalaré de manera esquemática algunas de las objeciones principales que se le pueden realizar, así como los problemas que tradicionalmente se han venido apuntando en torno a ella, en especial durante la recuperación del spinozismo en la filosofía francesa del siglo XX.

Primero, la comprensión del spinozismo como panteísmo -sea eleático o romántico- no está del todo justificada. Gueroult demostró que la cuestión en el spinozismo no es tanto que el todo (o el universo) se llame Dios, como que todo lo que existe lo hace "en" Dios ${ }^{5}$. Por lo tanto, la denominación más adecuada sería pan-en-teísmo.

Segundo, el reproche de Hegel al método matemático de demostración, afirmando que no era apto para la demostración de la verdad filosófica -un argumento que repite continuamente a lo largo de toda la obra- era, en principio, un reproche que también manejaba Spinoza ${ }^{6}$.

[5] Véase Gueroult 1968, 223; Lloyd 1996, 40. - Negri mantuvo que Spinoza propuso una «estrategia de constitución» contra la «utopía panteísta» (Negri 2000, 28-35).

[6] Deleuze sostuvo que Spinoza era completamente consciente de que el método de las matemáticas no era apropiado para tratar con el conocimiento filosófico (Deleuze 1990, 20). Es más, según su lectura, las esencias de los modos no son estructuras lógicas o matemáticas, sino res physicae (Deleuze 1990, 192). Finalmente, Deleuze señaló que esas nociones comunes que, a su juicio, constituyeron la conquista epistemológica esencial de la Ética de Spinoza, deben ser entendidas como «ideas biológicas en lugar de físicas o matemáticas» (Deleuze 1990, 278; véase también Deleuze 1988, 54-58). -La cuestión del método more geometrico (u ordine geometrico) de Spinoza fue explicada por Macherey como un concepto de la verdad estrictamente anti-cartesiano, un concepto de la verdad que, de hecho, estaba muy próximo al propio de Hegel. Para ambos, a pesar de que difieren en lo que cada uno atribuye al pensar, «la verdad es una determinación interna del pensamiento que excluye toda relación con un objeto exterior» (Macherey 2011, 73).- Lloyd nos ha puesto sobre aviso acerca de que la construcción estéril de la demostración 
Tercero, la definición de la causa de sí, que Hegel elogia como el momento del saber absoluto y explica como la determinación fundamental, principal, del sistema de Spinoza, en realidad no desempeña en este último la función de comienzo absoluto desde el que debe desarrollarse todo lo demás. Ser la causa de sí no es sino una propiedad de la substancia que meramente la explica; si la entendemos como definiendo o determinando la substancia, entonces ya habremos sometido la esencia de Dios a su poder y caído, por tanto, en la matriz del finalismo teológico ${ }^{7}$.

Cuarto, el spinozismo no se organiza ni como una filosofía del comienzo absoluto, ni como una filosofía del comienzo con el absoluto -al menos no en la Ética. Deleuze mostró la diferencia entre el Breve tratado sobre Dios, el hombre y su felicidad, que comienza en efecto con Dios, y la Ética, en la que la argumentación no empieza con Dios, sino que tiene como objeto que seamos capaces de ascender hacia Dios lo más rápido que podamos (Deleuze 1990, 76).

Quinto, hay muchos detalles que sugieren que el tratamiento que Hegel hizo de Spinoza fue más bien laxo, o incluso un intento deliberado de obligar al spinozismo a encajar en el espacio que Hegel le había preparado para la ocasión en el gran proyecto del desarrollo del espíritu a través de la historia de la filosofía. Para Macherey, un ejemplo abrumador de este modo de proceder era la reducción de la infinitud de atributos del sistema de Spinoza a solo dos, los de extensión y pensamiento (que luego explicaba como los "ser" y "pensar" básicamente eleáticos), una reducción que, al parecer, no sirve a otro propósito que el de situar a Spinoza inmediatamente después de Descartes en la secuencia lógico-histórica de la filosofía (Macherey 1992, 149).

Pero, ya en sexto lugar, ninguno de los otros aspectos de la lectura de Spinoza que hace Hegel gozó de tan largo recorrido como el célebre principio de omnis determinatio est negatio (TWA20, 164). No fue un invento de Hegel: le llegó en relación a Spinoza por medio de Jacobi. La frase estaba en el ambiente incluso antes de Jacobi, pero fue él quien le dio la forma de un principio ontológico, según el cual todas las cosas determinadas están marcadas por una decadencia intrínseca (negación) (Düsing 1992, 183). Pero, si seguimos el análisis de Macherey, la asunción de este principio por parte de Hegel es aún más fascinante, puesto que lo leyó a la inversa: como si toda negación fuese determinación, es decir, como si la negación de un ente fuese, de hecho, un procedimiento productivo de determinación (positiva) (Macherey 2011, 115). El gesto hegeliano hacia el spinozismo puede resumirse, entonces, de este modo: si Spinoza hubiera comprendido "su" principio como un principio especulativo,

matemática en Spinoza puede resultar engañosa, en tanto que el libro está repleto de ironías e ingenio e, incluso, de comentarios emocionales (Lloyd 1996, 19-20).

[7] Gueroult 1968, 41; Macherey 2011, 16. 
esto es, como un principio de potencialidad, entonces su sistema de una substancia absolutamente infinita y modos finitos jamás habría sido solo un sistema de degradación ontológica, de simple negación, sino un sistema de negación de la negación, de negación productiva.

Sin embargo, el principio de degradación ontológica no es un principio spinoziano en absoluto. Hegel entendió el sistema de la substancia, los atributos y los modos como si fuera un sistema emanacionista típico (Düsing 1992, 169-171). Ahora bien, pese a que Spinoza, en efecto, se había inspirado en la extensa tradición neoplatónica de la causalidad emanacionista, Deleuze ha recalcado que este produjo en su interior un importante giro inmanentista. Tanto la causa emanativa como la inmanente permanecen en ellas mismas cuando producen su efecto -pero difieren la una de la otra en que, en el caso de la causa inmanente, el efecto también "permanece" en su causa. Esta distinción tiene enormes consecuencias para todo el sistema porque la causa inmanente no conoce ninguna jerarquía ontológica, y en tanto que el efecto no llega a "desprenderse" de su causa, tampoco necesita "regresar" teleológicamente a ella (Deleuze 1990, 171-174).

Y como si todo esto no fuera suficiente: algunos de los reproches de Hegel a Spinoza no solo no parecen estar justificados, sino que ven sin necesidad un adversario donde en realidad hay un aliado. El ejemplo más palmario de ello es la cuestión del método. El famoso ejemplo del hierro forjado del Tratado de la reforma del entendimiento de Spinoza es un ataque al método de Descartes de la percepción clara y distinta: si se admite la premisa de que para forjar el hierro uno debe adquirir antes las herramientas adecuadas, entonces también debe aceptarse necesariamente que para producir esas herramientas uno debe adquirir antes otras herramientas y así ad infinitum (Spinoza 1988, 85). La definición de una idea adecuada que Spinoza introduce en la Ética deja bastante claro que se trata de una idea que "en cuanto considerada en sí misma, sin relación al objeto, posee todas las propiedades o denominaciones intrínsecas de una idea verdadera" (Spinoza 2011, 122). Por tanto, el concepto de "método" de Spinoza exige una relación intrínseca con la verdad, que es justo lo que Hegel sostenía con tanta brillantez en la introducción de la Fenomenología del espíritu, cuando criticaba la exigencia de Kant de que uno debe discutir los límites del conocimiento antes de intentar alcanzar la verdad (Fen. 143). Todo esto parece sugerir que eso que a Hegel tanto le entusiasmaba, y al mismo tiempo le irritaba, del spinozismo era... Hegel mismo. 


\section{El SABER ABSOLUto EN EL MATERIALISMO FRANCÉS DEL SIGLO XX}

Y puede que todo lo anterior sea aplicable a la imagen de Spinoza en el idealismo alemán en general: lo necesitaban precisamente por ser la imagen frente a la cual podían formular sus propias posiciones filosóficas ${ }^{8}$. No obstante, quizás se puede decir algo parecido sobre la imagen de Hegel en lo que podríamos llamar materialismo francés del siglo XX. En Althusser, no solo podemos detectar la tendencia estereotipante que reduce a Hegel a una colección de saberes vacíos, sino también el fracaso a la hora de percibir la proximidad de algunas de sus propias empresas filosóficas a las de Hegel. Por añadidura, es importante darse cuenta de que Althusser, en su oposición a Hegel, se apoyó con fuerza en la filosofía de Spinoza.

Recordemos la versión de Althusser acerca de qué era lo que estaba en juego en la inversión materialista de la dialéctica hegeliana. Para Althusser, no se trata solo de tomar oposiciones binarias tales como materia/idea, práctica/ teoría, economía/ideología y luego dar la vuelta al orden de prioridad. En su lugar, reclama la renuncia total al modelo jerárquico ontológico-causal de la determinación. Semejante modelo era característico de los sistemas metafísicos del Neoplatonismo, y Althusser apoyó enérgicamente el principio spinozista de - por recurrir a la terminología de Deleuze- la univocidad del ser9.

Este principio es la clave para entender las tesis de Althusser, en las cuales la teoría no es lo opuesto de la práctica, sino que es tal o cual práctica teórica; en las cuales la ideología del estado no es lo opuesto a la producción económica, sino que es tal o cual producción ideológica. Esto ya determina la imagen de Hegel que Althusser rechaza: es la imagen de Hegel como inversión del Neoplatonismo. Macherey lo explica así: "lo que propone Hegel es simplemente invertir este orden situando el todo al final del proceso y organizando sus determinaciones como momentos que conducen progresivamente hacia allí" (Macherey 2011, 75). Lo que los althuserianos vieron en Hegel fue la encarnación tanto de la teología como de la teleología, a la que se añadía el modelo ontológico de los sistemas de la causalidad emanativa, en el cual, en lugar del descenso del absoluto para regresar ulteriormente a él, tenemos el proceso de cancelación, de Aufhebung, como avance constante hacia él. Esta es la razón por la que daban cuenta del vilipendiado concepto del saber absoluto como el punto mítico en el que convergían el conocimiento y la verdad que, de este

[8] Tavoillot lo expresó de la mejor manera: «Savoir ce que Spinoza a vraiment dit importe alors moins que de savoir si ce qu'il a dit est vrai» (Tavoillot 2007, 36-37).

[9] Para Deleuze, Scoto fue el precursor filosófico esencial de Spinoza. Véase especialmente: Deleuze 1990, 58-67. 
modo, "rumia sordamente aún en los fantasmas religiosos de la epifanía del adviento" (Althusser 1970, 16).

Uno debe notar que Althusser y sus alumnos no fueron la única escuela francesa que vio a la teleología y a la teología caminando de la mano a través de Hegel. Derrida señaló que Lacan, Althusser, Foucault, Deleuze, Sartre, MerleauPonty, Lévinas y muchos otros experimentaron un caso de "alergia organizada hacia la dialéctica hegeliana", cada uno con sus respectivos trasfondos teóricos y puntos de entrada (Derrida 2005, xxvi). Todo ello empezó con las legendarias conferencias de Koyré y Kojève, cuyas lecturas influyeron enormemente en varias generaciones de académicos y pensadores. Catherine Malabou señala que la lectura del saber absoluto como fin de los tiempos que hizo Kojève, la cual sentó los cimientos de la idea del saber absoluto como convergencia de todas las oposiciones, estaba fuertemente influida por la explicación de Heidegger del saber absoluto como parusía y su afirmación de que el tiempo, para Hegel, era primariamente el tiempo pasado (Malabou 2005, 3). Se podría afirmar entonces que una gran parte del rechazo francés a Hegel está basado en la crítica de Heidegger al hegelianismo en tanto metafísica onto-teológica.

Por descontado, es verdad que, en la construcción de su Fenomenología del espíritu, Hegel puso el capítulo sobre el saber absoluto inmediatamente después del capítulo sobre la religión revelada; de hecho, lo puso como la conclusión lógica de ese capítulo. El espíritu de la religión revelada todavía está para sí separado de su objeto, pese a que claramente ambos son uno en sí. La conciencia aún no se ha reconciliado o reunido con la esencia. Hegel escribe: "Por eso, su propia reconciliación hace entrada como algo lejano en su consciencia, algo lejano del futuro" (Fen. 893). Esta es la manera en la que el propio Hegel formula la necesidad del advenimiento del saber absoluto; la referencia a la parusía como la reconciliación futura es evidente.

Pero, a pesar de todo, el conocimiento que está en juego en el saber absoluto no es el de alguna verdad místico-religiosa. En el saber absoluto, la conciencia no aprende nada nuevo. El saber absoluto no es la eliminación mítica de la diferencia entre el sujeto y el objeto, entre la verdad y el conocimiento, no es el Santo Grial del conocimiento, el momento profetizado de una verdad inmediata y definitiva en la que unas palabras concretas expresan un ser concreto. En su lugar, el saber absoluto es un punto vacío. Es justamente el concepto de la irreconcialibilidad fundamental que está en el corazón de la verdad misma. Por utilizar una expresión recurrente propuesta por Mladen Dolar: la verdad no es sino el hiato entre la verdad y el conocimiento (Dolar 1992, 9-10). El saber absoluto es el lugar de este vacío, y este vacío es lo que produce el efecto de la parusía. 
Quizás podríamos decir que el saber absoluto funciona como un signo de puntuación al final de una frase. La puntuación misma no tiene contenido; es simplemente una decisión formal de que el proceso ha llegado a su fin. De este modo, remite al lector de vuelta a la frase misma, produciendo el efecto del significado que había estado en la frase desde el principio.

Los signos de puntuación pertenecen claramente al campo de la escritura. En la medida en que no hay sonido para ellos, podría parecer que le son impuestos al lenguaje hablado natural, orgánico. Sin embargo, forma parte del conocimiento lingüístico ordinario que tal imposición es, de hecho, característica del lenguaje mismo. Para Saussure, lo que los signos están significando no se define por su contenido positivo o afirmativo, sino que se define "negativamente por sus relaciones con otros términos del sistema" y, por tanto, "su característica más peculiar radica en ser lo que otros no son" (Saussure 1959, 117). Para Derrida, la negatividad de la escritura, su necesaria dilación y deformación se encuentra, de hecho, inscrita en la esencia del lenguaje mismo. Él escribe: "La propia idea de la institución [...] del signo es impensable antes de la posibilidad de la escritura y fuera de su horizonte" (Derrida 1997, 44). La negatividad del lenguaje implica que el signo de puntuación es, de hecho, el fenómeno primordial del lenguaje, su más íntima posibilidad, su propia índole.

El saber absoluto opera precisamente con esta doble función. Por un lado, funciona como la detención total al final de la frase, como el punto del Juicio Final prometido; pero, por otro lado, este Juicio Final termina siendo puramente vacío y sin dimensión, nada más que un punto formal que nos revela que todo contenido positivo, afirmativo, de un Juicio Final siempre es un efecto o un producto de su proceso. Este gesto formal del signo de puntuación es radicalmente ajeno a Spinoza y a las encarnaciones contemporáneas del spinozismo. Este simple gesto era justo lo que Hegel, in nuce, echaba de menos en Spinoza: lo que Spinoza dijo era todo lo que tenía que ser dicho, lo único que le faltaba era el signo de puntuación al final de su frase.

\section{LA CUESTIÓN DE LA NEGATIVIDAD}

Se podría realizar la observación general de que la relación entre el hegelianismo y el spinozismo es, en muchas ocasiones, una relación de fascinación mutua en la cual cada uno está fascinado consigo mismo en el otro. Parece que Hegel, y el idealismo alemán en general, vieron en Spinoza una imagen que les ayudaba a formular mejor sus propios proyectos, pero su lectura, por desgracia, tenía poco que ver con la filosofía spinoziana misma. Al mismo tiempo, se podría afirmar que los spinozianos franceses -Althusser y Deleuze- vieron en Hegel la caricatura de un teólogo y un finalista a quien detestaban y contra 
el que se querellaban con regocijo, pero fracasaron a la hora de ver el sustrato común que unía sus proyectos al de Hegel.

Si tratamos de recapitular los tres aspectos en que los materialistas franceses criticaron a Hegel, obtenemos un cuadro fascinante: lo hicieron por las mismas razones por las que Hegel y los idealistas alemanes criticaron a Spinoza. En primer lugar, nos encontramos con el argumento de que el proyecto hegeliano es una inversión de la filosofía de la emanación; esto refleja exactamente lo que Hegel estaba criticando en Spinoza. En segundo lugar, tanto Althusser como Deleuze criticaron a Hegel por su concepto de contradicción; afirmaron que se trataba de cierta suerte de dispositivo lógico que había sido inventado para reintroducir, por la puerta de atrás, la filosofía de la identidad. De nuevo, Hegel había criticado al spinozismo por la misma razón, a saber, por ser un sistema de la identidad, incapaz de pensar la contradicción. Y, en tercer lugar, criticaron a Hegel por presentar un sistema que no podía hacerse cargo de sorpresas o accidentes genuinos, en tanto que su proceso dialéctico parece avanzar en pasos lógicos y predecibles hacia un final lógico y predecible. Aquí volvemos a oír el eco de la crítica de Hegel a Spinoza como un determinismo rígido.

Estas objeciones -determinismo, teleología mecánica, funcionalismo- pasan por alto que tanto los hegelianos como los spinozianos, tal y como hicieron Hegel y Spinoza, argumentan a favor de una substancia capaz de organizar su propia transformación. Cómo demostrar exactamente esa autodeterminación, en virtud de qué razones se puede sostener y qué conceptos y estrategias concretas se han de emplear - esta es la cuestión de Hegel y Spinoza. La problemática de la recepción apunta, a su manera, a la cuestión del movimiento dentro del absoluto, a la cuestión del estatuto contradictorio del comienzo y la cuestión de un dinamismo irreducible, oculto en el paisaje positivo de un texto. Da la impresión de que la lucha titánica entre la crítica hegeliana de Spinoza, por un lado, y la crítica spinoziana de Hegel, por el otro, se libra en tres frentes: el del rechazo al concepto metafísico de la identidad, el del rechazo a la ontología emanacionista y el de la defensa del concepto de lo accidental o aleatorio. Lo que estos tres frentes tienen en común puede resumirse en la cuestión de la negatividad. Parece que la cuestión de la negatividad, que despierta un interés creciente en los debates contemporáneos, es en muchos sentidos una repetición productiva de la cuestión que definió el campo de batalla principal del idealismo alemán. Podemos oír el eco de estas luchas tectónicas en las controversias entre los deleuzianos y los lacanianos de nuestros días ${ }^{10}$.

[10] Adrian Johnston escribe: «Me atrevería a mantener que uno de los antagonismos principales que dividen hoy en día al materialismo desde su interior es el que existe entre la corriente neospinoziana y la neohegeliana. La primera (encarnada, por ejemplo, por Louis Althusser, Gilles Deleuze y su diversa progenie) intenta disolver la figura del sujeto, mientras que 
Concluiré con una observación final. Pese a que está claro que la cuestión de la negatividad productiva es lo que estaba en juego para Hegel cuando criticaba a Spinoza, no está tan claro cómo podemos ocuparnos de la cuestión de la negatividad en Spinoza o en los spinozianos contemporáneos, como Deleuze. Si sus filosofías se basan en algo, es en la idea de un concepto afirmativo de producción. Sin embargo, la substancia spinoziana debe ser capaz de autodeterminación, debe ser posible verla como una substancia "plástica", por decirlo así, pues, de otro modo, sería vulnerable, en efecto, a la objeción de ser un determinismo ciego. Esta plasticidad de la substancia misma es lo que podríamos llamar, en Spinoza, negatividad.

\section{REFERENCIAS BIBLIOGRÁFICAS}

L. Althusser, E. Balibar: Reading Capital, tr. B. Brewster, Londres, Verso, 1970.

G. Deleuze: Spinoza: Practical Philosophy, tr. R. Hurley, San Francisco, City Light Books, 1988.

G. Deleuze: Expressionism in Philosophy: Spinoza, tr. M. Joughin, Nueva York, Zone Books, 1990.

J. DeRrida: «A time for farewells: Heidegger (read by) Hegel (read by) Malabou» en C. Malabou: The Future of Hegel, Londres/Nueva York, Routledge, 2005.

J. DeRrida: Of Grammatology, tr. G. Chakravorty Spivak, Baltimore, John Hopkins University Press, 1997.

M. Dolar: Heglova Fenomenologija duha I. Ljubljana: Društvo za teoretsko psihoanalizo, 1990.

K. DüsING: «Von der Substanz zum Subjekt. Hegels spekulative Spinoza-Deutung» en M. Whalter (ed.): Spinoza und der Deutsche Idealismus, Wurzburgo, Köningshausen und Neumann, 1992, pp. 163-180.

H-G. Gadamer: Truth and Method, tr. J. Weinsheimer y D.G. Marshall, Londres/ Nueva York, Continuum, 2004.

M. Gueroult: Spinoza I. Dieu. (Ethique, I). Paris, Aubier-Montaigne, 1968.

G. W. F. Hegel: Ciencia de la lógica, edición de Félix Duque, Madrid, Abada, 2011.

G. W. F. Hegel: Fenomenología del espíritu, edición bilingüe de Antonio Gómez Ramos, Madrid, Abada, 2010.

G. W. F. Hegel: Werke in zwanzig Bänden, (eds.) E. Moldenhaus y K. M. Michel,

la segunda (representada principalmente por Žižek y el lacanismo esloveno) intenta preservarlo» (Johnston 2014, 20). 
Fráncfort del Meno, Suhrkamp, 1970.

A. Johnston: Adventures in Transcendental Materialism. Dialogues with Contemporary Thinkers, Edimburgo, Edinburgh University Press, 2014.

G. Lloyd: Spinoza and the Ethics, Londres/Nueva York, Routledge, 1996.

P. Macherey: Hegel or Spinoza, tr. Susan B. Ruddick. Minneapolis, University of Minnesota Press, 2011.

C. Malabou: The Future of Hegel, Londres/Nueva York, Routledge, 2005.

A. Negri: The Savage Anomaly, tr. M. Hardt, Minneapolis, University of Minnesota Press, 2000.

F. SAussure: Course in General Linguistics, tr. W. Baskin, Nueva York/Toronto/Londres, McGraw Hill, 1959.

B. Spinoza: Ética demostrada según el orden geométrico, tr. Vidal Peña, Madrid, Alianza, 2011.

B. Spinoza: Tratado de la reforma del entendimiento. Principios de la filosofía de Descartes. Pensamientos metafísicos, tr. A. Domínguez, Madrid, Alianza, 1988.

P-H. Tavoillot: «Spinoza dans la querelle du panthéisme» en A. Tosel, P-F. Moureau y J. Salem (eds.): Spinoza au XIXe siècle, Paris, Publications de la Sorbonne, 2007, pp. 35-45.

J-M. VAysse: «Spinoza dans la problématique de l'idealisme allemande» en A. Tosel, P-F. Moureau y J. Salem (eds.): Spinoza au XIXe siècle, Paris, Publications de la Sorbonne, 2007, pp. 65-74.

S. ŽıžEK: The Fragile Absolute, Londres/Nueva York, Verso, 2001.

Dr. Gregor Moder is Assistant Professor at the University of Ljubljana,

\section{AdDress:}

Faculty of Arts

Department of Philosophy

Aškerčeva 2

1000 Ljubljana, Slovenia

e-mail: gregor.moder@ff.uni-lj.si 
RESEARCH INTERESTS:

German Idealism, Theory of Ideology, Philosophy of Art, Philosophy of Culture

SELeCted Bibliography since $2013\left({ }^{*}=\right.$ translation $)$

Books

G. Moder: Hegel and Spinoza. Substance and Negativity. Evanston, IL, Northwestern University Press, forthcoming in July 2017. [new, reworked and updated edition]

${ }^{\star}$ G. Moder: Hegel und Spinoza. Negativität in der gegenwärtigen Philosophie, tr. A. Leskovec, Vienna/Berlin, Turia+Kant, 2012 [2013].

G. Moder: Komična ljubezen. Shakespeare, Hegel, Lacan. Ljubljana, DTP, 2016

JOURNAL ARTICLES, BOOK CONTRIBUTIONS

G. Moder: "What can Althusser Teach us about Street Theater and Vice Versa" in Stasis, No. 1, 2014.

G. Moder: "The Beard, the Bust and the plumed Helmet” in I. Novak, J. Krečič, M. Dolar (eds.): Lubitsch Can't Wait, Ljubljana, Slovenska kinoteka, 2014.

G. Moder: “After Catastrophe. From Beckett to Žižek” in S Journal, Vol. 6, 2014.

G. Moder: “'Held out into the nothingness of being:' Heidegger and the Grim Reaper” in Filozofski vestnik, No. 2, 2013.

${ }^{\star}$ G. Moder: "Der Bart, die Büste und der gefiederte Helm" in Neue Rundschau, Frankfurt am Main, S. Fischer, No. 3, 2013.

G. Moder: "Comedy and Negativity" in Stasis, No. 1, 2013. 